\section{Current approaches to the treatment of non-Hodgkin's lymphoma}

\author{
Christian Gisselbrecht \\ Hôpital Saint Louis, Paris, France
}

\section{Abstract}

Chemotherapy with cyclophosphamide, doxorubicin, vincristine, and prednisone (CHOP) has long been a standard treatment for lymphoma. Improvements to the efficacy of this regimen can be made by increasing the doses of doxorubicin and cyclophosphamide, as in the chemotherapeutic regimen of doxorubicin, cyclophosphamide, vindesine, bleomycin, and prednisone (ACVBP), and by reducing the standard dosing interval, as seen with the CHOP-14 regimen. Adding the immunotherapeutic agent rit uximab (R) to either CHOP or ACVBP has been shown to improve outcomes significantly, such that six cycles of R-CHOP plus two cycles of rituximab are as effective as eight cycles of R-CHOP, and R-CHOP-21 appears to be at least as effective as the more dose-intense R-CHOP-14. In patients who have several adverse prognostic factors, RACVBP plus autologous stem-cell transplantation has been shown to produce good treatment outcomes. The use of positron emission tomography scanning before and early in treatment should allow prediction of long-term outcomes, and therefore the adaptation of treatment to individual prognosis and treatment needs. In patients with follicular lymphoma, rituximab has been shown to improve the efficacy of conventional chemotherapies. In addition, rituximab alone or yttrium-90-ibritumomab tiuxetan are effective maintenance therapies in this condition.

\section{Introduction}

There are now about 90 defined subtypes of lymphomas, and the clinical outcomes are different for the different subtypes, although many cannot currently be easily distinguished. ${ }^{1}$ Initially, lymphomas were classified by clinical factors, which allowed clinicians to distinguish those that had a good prognosis from those that had a poor prognosis. More recently, molecular signatures associated with prognosis have been identified.2 For example, the germinal center B-cell-like (GCB) subtype of diffuse large B-cell lymphoma (DLBCL) was found to have a significantly better survival rate than the non-GCB DLBCL. However, such gene profiling is not yet possible as a clinical routine, so the search is ongoing for a clinical surrogate to identify these subtypes.

\section{Improving on the efficacy of $\mathrm{CHOP}$}

The standard regimen of cyclophosphamide, doxorubicin, vincristine, and prednisone (CHOP) given over a 3-weekly cycle (CHOP21) was easy to use and produced acceptable response rates. However, it did not provide a long-term survival benefit for most patients only about $40 \%$ of patients had long-term disease control. ${ }^{3}$ Several attempts were therefore made to improve on the efficacy of this regimen, using conventional chemotherapy.

Treatment with doxorubicin, cyclophosphamide, vindesine, bleomycin, and prednisone (ACVBP)-14, in which the doses of both doxorubicin and cyclophosphamide are higher than in the standard CHOP regimen, was originally devised as a standard treatment for all Tcell lymphomas. In two studies, there was a significant improvement in favor of ACVBP compared with CHOP-21, in patients who were older and had a poor prognosis, ${ }^{4}$ and in those who were young and had a good prognosis, ${ }^{5} \mathrm{al}-$ though ACVBP was more toxic than CHOP. In a third study, ACVBP was shown to be more effective than methotrexate, bleomycin, doxorubicin, cyclophosphamide, vincristine, and dexamethasone (m-BACOD) in patients with lowrisk aggressive lymphoma. ${ }^{6}$

Increasing the dose intensity of $\mathrm{CHOP}$ by reducing the dose interval from 3 to 2 weeks (CHOP-14) produced a clear increase in efficacy in both young and elderly patients. ${ }^{7,8} \mathrm{An}$ other study investigated the efficacy of ACVBP followed by either sequential high-dose chemotherapy or intensive chemotherapy plus autologous bone marrow transplant, in patients with a high International Prognostic Index (IPI). Bone marrow transplant was associated with $64 \%$ overall survival in the long term. ${ }^{9}$ Thus, this was the level of response and efficacy that could be produced in patients with a poor prognosis, prior to the introduction of rituximab therapy.

\section{Treatment with R-CHOP}

The first demonstration that rituximab plus CHOP (R-CHOP) was more effective than CHOP alone was obtained after the initial 2 years of the Groupe d'Étude des Lymphomes de l'Adulte (GELA) LNH98.5 study.10 Long-term follow-up of this study over 7 years (and now 10 years) has shown that the advantages are maintained, in terms of event-free, progression-free, disease-free, and overall survival.11 This superiority of R-CHOP over CHOP was subsequently confirmed in other studies: the MInT study in young patients with good prognosis, 12 the RICOVER study in elderly patients, ${ }^{13}$ the British Columbia study in patients with advanced disease, ${ }^{14}$ and the Eastern Cooperative Oncology Group study in elderly patients, which also demonstrated the efficacy of rituximab mainte-
Correspondence: Professor Christian Gisselbrecht, Institut d'Hematologie, Hôpital Saint Louis, 1 avenue Claude Vellefaux, 75010 Paris, France. Tel. +33.1.4249.4190 - Fax: +33.1.4249.9972 E-mail: christian.gisselbrecht@gmail.com

Key words: non-Hodgkin's lymphoma, treatment, chemotherapy, rituximab.

Conflict of interest: the author has received research funding from Roche and Bayer Schering Pharma.

This work is licensed under a Creative Commons Attribution NonCommercial 3.0 License (CC BYNC 3.0).

CC Copyright C. Gisselbrecht, 2011

Licensee PAGEPress, Italy

Hematology Reports 2011; 3(s3):e3

doi:10.4081/hr.2011.s3.e

nance therapy. 15 The RICOVER study also showed that six cycles of CHOP-14 plus eight infusions of rituximab were as effective as eight cycles of R-CHOP-14.13

A final research question remained about the relative efficacies of the 2-versus 3 -week $\mathrm{R}-\mathrm{CHOP}$ regimens (R-CHOP-14 versus $\mathrm{R}$ CHOP-21), as these had not been compared directly. The interim results of the $\mathrm{LNH} 03-6 \mathrm{~B}$ study have been presented in abstract form, ${ }^{16}$ and showed no significant difference between the two regimens, and a somewhat higher efficacy and lower toxicity for the less dose-intense R-CHOP-21. A second interim analysis, recently presented at the $11^{\text {th }}$ International Conference on Malignant Lymphoma, confirmed these results. 17

\section{Improving on the efficacy of $\mathrm{R}-\mathrm{CHOP}$}

The efficacies of R-ACVBP and R-CHOP were compared in the LNH03-2B study (clinicaltrials.gov identifier NCT00140595), which involved young patients with DLBCL and an ageadjusted IPI of 1. An interim analysis showed superiority of R-ACVBP-14 over R-CHOP-21 in terms of 2-year event-free survival. The final analysis, recently presented at the $52^{\text {nd }}$ American Society of Hematology Annual Meeting in December 2010, confirmed a significant advantage for R-ACVBP at 3 years in terms of eventfree, progression-free, disease-free, and overall survival.18

There is little evidence for how best to treat patients with 2-3 adverse prognostic factors, although historically outcomes have been poor. A retrospective analysis of patients with DLBCL treated with R-CHOP showed that response rates were good for patients with 0-2 adverse prognostic factors, but 4-year progression-free survival and overall survival rates were only just over $50 \%$ for patients with more than two adverse 
prognostic factors. 19 A group of these hard-totreat patients (with IPIs of 2-3) were given four cycles of R-ACVBP, followed by carmustine, etoposide, cytarabine, and melphalan (BEAM) and autologous stem-cell transplantation..$^{20}$ After 4 years of follow-up, progression-free survival was high at $76 \%$ (Figure 1), and there was no difference in outcomes between groups of patients with two or three adverse prognostic factors. ${ }^{20}$ In order to compare ACVBP regimens with and without rituximab, a matched control study was conducted between two trials (LNH98-3B and LNH03-3B), which showed a large difference in favor of the rituximab-containing regimen..$^{20}$ The results indicate that treatment efficacy can be improved for these patients with IPIs of 2-3, and that an intensive treatment regimen may provide them with a probability of about $75 \%$ of living without relapse.

\section{Adapting treatment to individual patient risk}

An initial study of the use of [18F]fluoro-2deoxy-D-glucose positron emission tomography (PET) before treatment, after two cycles, and after four cycles showed that it was possible to identify those patients who were PET-negative early in treatment, and thereby to predict with a high degree of accuracy those who would remain diseasefree (Figure 2).21 Further studies have refined these assessment methods, to reduce the incidence of false-positives and help develop standardized techniques and criteria that can be widely used.22 Based on these initial results, a Phase II study (LNH2007-3B) has been started to follow PET changes under two treatment regimens, namely R-CHOP-14 and R-ACVBP-14, in patients with DLBCL and an age-adjusted IPI of 2-3.23 PET scans will be performed at baseline, and after two and four cycles, and treatment adapted after cycle 4 depending on the PET results.

\section{Treatment of follicular lymphoma}

Follicular lymphoma usually grows and spreads slowly, and has few symptoms. The current goal of therapy is to maintain the best quality of life for patients and to treat them only when they develop symptoms. Any therapeutic approach should demonstrate an improvement in patient survival but, because median survival is $14-15$ years, surrogate markers such as progression-free survival are needed to assess possible survival benefits.

Randomized trials clearly demonstrated that the addition of rituximab to the commonly used chemotherapies increased patient response rates and survival.24-27 Therefore, these patients should receive rituximab in addition to chemotherapy. Two studies have also investigated the effects of maintenance therapy in patients who responded to initial treatment. One reported the use of yttrium-90-ibritumomab tiuxetan versus no further treatment for patients who showed a complete or partial remission, and showed an advantage for maintenance treatment over the watch-and-wait strategy. ${ }^{28}$ A second study (PRIMA), completed in 2007 , included 1193 patients, $74 \%$ of whom had received previous $\mathrm{R}-\mathrm{CHOP}$ and $23 \%$ of whom had received previous rituximab, cyclophosphamide, vincristine, and prednisone (R-CVP). ${ }^{27}$ The results were in favor of rituximab maintenance therapy, compared with observation alone. After a median follow-up of 36 months, progression-free survival was $74.9 \%$ among those receiving rituximab versus $57.6 \%$ in the observation group $(\mathrm{P}<0.0001) .27$

\section{Conclusions}

CHOP has long been the standard therapy for lymphoma. Improvements have been made to the efficacy of this regimen by increasing the dosing frequency, increasing the doses (in the ACVBP regimen), and by adding rituximab immunotherapy to either of these regimens. Research is now underway to investigate the place of new therapies such as lenalidomide, new immunotherapies, and high-dose rituximab in improving patients' overall survival (see Burchardt in this supplement).

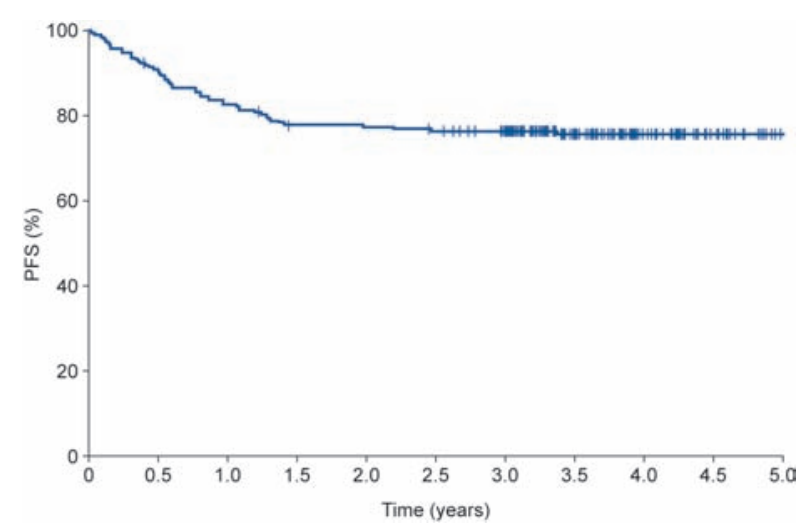

Figure 1. Progression-free survival (PFS) in 208 hardto-treat patients (with an International Prognostic Index of 2-3) with diffuse large B-cell lymphoma, who were given four cycles of rituximab, doxorubicin, cyclophosphamide, vindesine, bleomycin, and prednisone (R-ACVBP), followed by carmustine, etoposide, cytarabine, and melphalan (BEAM) and autologous stem-cell transplantation (unpublished data). Intention-to-treat population.

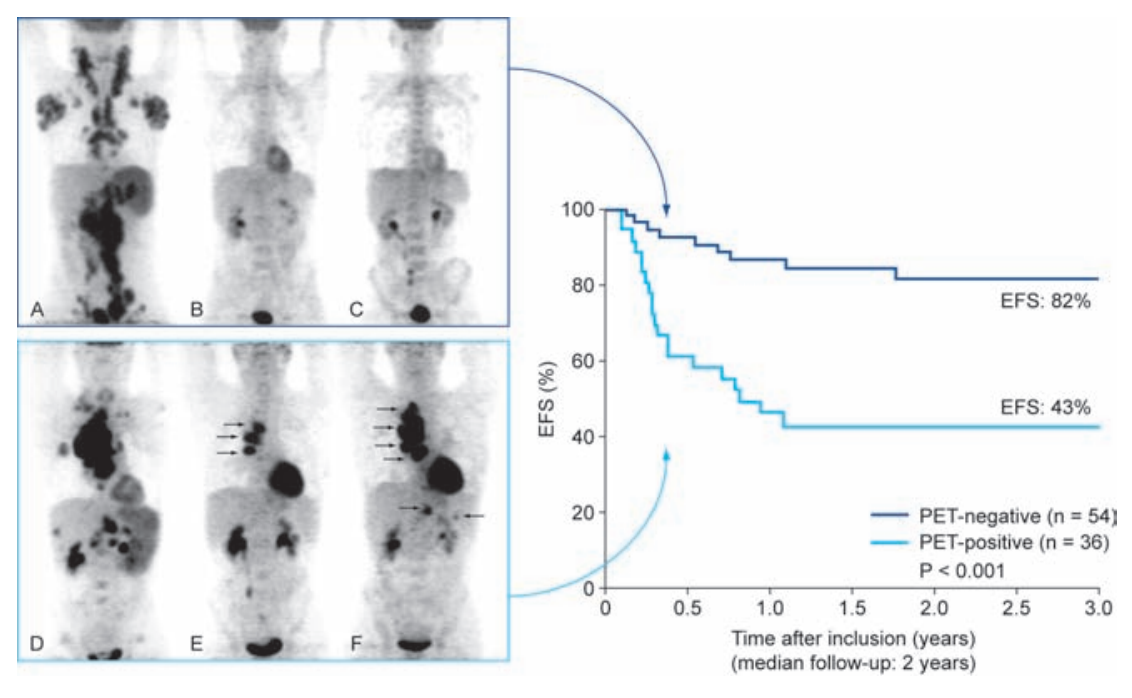

Figure 2. Examples of sequential [18F]fluoro-2-deoxy-D-glucose positron emission tomography (PET) findings in two patients. ${ }^{21}$ Panels A-C show scans from a patient with truly negative early PET, predicting complete response. Panels D-F show scans from a patient with truly positive early PET, predicting relapse. Arrows show hilar foci after two (panel E) and four (panel F) cycles of chemotherapy. The Kaplan-Meier plot shows 2-year estimates of event-free survival (EFS) according to early PET status after two cycles of chemotherapy. ${ }^{21}$ From Haioun C et al. [18F] fluoro-2-deoxy-D-glucose positron emission tomography (FDG-PET) in aggressive lymphoma: an early prognostic tool for predicting patient outcome. Blood 2005;106:1376-81. Reprinted with permission from the American Society of Hematology via the Copyright Clearance Center and the authors. 


\section{References}

1. Jaffe ES, Harris NL, Stein H, Isaacson PG. Classification of lymphoid neoplasms: the microscope as a tool for disease discovery. Blood 2008;112:4384-99.

2. Hans CP, Weisenburger DD, Greiner TC, et al. Confirmation of the molecular classification of diffuse large B-cell lymphoma by immunohistochemistry using a tissue microarray. Blood 2004;103:275-82.

3. Fisher RI, Gaynor ER, Dahlberg S, et al. Comparison of a standard regimen (CHOP) with three intensive chemotherapy regimens for advanced non-Hodgkin's lymphoma. N Engl J Med 1993;328:1002-6.

4. Tilly $\mathrm{H}$, Lepage E, Coiffier B, et al. Intensive conventional chemotherapy (ACVBP regimen) compared with standard CHOP for poor-prognosis aggressive non-Hodgkin lymphoma. Blood 2003;102:4284-9.

5. Reyes F, Lepage E, Ganem G, et al. ACVBP versus CHOP plus radiotherapy for localized aggressive lymphoma. N Engl J Med 2005;352:1197-205.

6. Tilly H, Mounier N, Lederlin P, et al. Randomized comparison of ACVBP and mBACOD in the treatment of patients with low-risk aggressive lymphoma: the LNH871 study. Groupe d'Etudes des Lymphomes de l'Adulte. J Clin Oncol 2000;18:1309-15.

7. Pfreundschuh M, Trumper L, Kloess M, et al. Two-weekly or 3-weekly CHOP chemotherapy with or without etoposide for the treatment of elderly patients with aggressive lymphomas: results of the NHL-B2 trial of the DSHNHL. Blood 2004;104:634-41.

8. Pfreundschuh M, Trumper L, Kloess M, et al. Two-weekly or 3-weekly CHOP chemotherapy with or without etoposide for the treatment of young patients with good-prognosis (normal LDH) aggressive lymphomas: results of the NHL-B1 trial of the DSHNHL. Blood 2004;104:626-33.

9. Haioun C, Lepage E, Gisselbrecht C, et al. Survival benefit of high-dose therapy in poorrisk aggressive non-Hodgkin's lymphoma: final analysis of the prospective LNH87-2 protocol - a groupe d'Etude des lymphomes de l'Adulte study. J Clin Oncol 2000;18:3025-30.

10. Coiffier B, Lepage E, Briere J, et al. CHOP chemotherapy plus rituximab compared with $\mathrm{CHOP}$ alone in elderly patients with diffuse large-B-cell lymphoma. $\mathrm{N}$ Engl $\mathrm{J}$ Med 2002;346:235-42.

11. Coiffier B, Feugier P, Mounier N, et al. Long-term results of the GELA study com- paring R-CHOP and CHOP chemotherapy in older patients with diffuse large B-cell lymphoma show good survival in poor-risk patients. J Clin Oncol 2007;25:Abs 8009.

12. Pfreundschuh M, Trumper L, Osterborg A, et al. CHOP-like chemotherapy plus rituximab versus CHOP-like chemotherapy alone in young patients with good-prognosis diffuse large-B-cell lymphoma: a randomised controlled trial by the MabThera International Trial (MInT) Group. Lancet Oncol 2006;7:379-91.

13. Pfreundschuh M, Schubert J, Ziepert M, et al. Six versus eight cycles of bi-weekly CHOP-14 with or without rituximab in elderly patients with aggressive CD20+ B-cell lymphomas: a randomised controlled trial (RICOVER-60). Lancet Oncol 2008;9:105-16.

14. Sehn LH, Donaldson J, Chhanabhai M, et al. Introduction of combined CHOP plus rituximab therapy dramatically improved outcome of diffuse large B-cell lymphoma in British Columbia. J Clin Oncol 2005;23:5027-33.

15. Habermann TM, Weller EA, Morrison VA, et al. Rituximab-CHOP versus CHOP alone or with maintenance rituximab in older patients with diffuse large B-cell lymphoma. J Clin Oncol 2006;24:3121-7.

16. Delarue R, Tilly H, Salles G, et al. R-CHOP14 compared to R-CHOP21 in elderly patients with diffuse large B-cell lymphoma: results of the interim analysis of the LNH03-6B GELA study. Blood 2009;114:Abs 406.

17. Delarue R, Tilly H, Salles G, et al. R-CHOP14 compared to R-CHOP21 in elderly patients with diffuse large B-cell lymphoma (DLBCL): results of the second interim analysis of the LNH03-6B GELA study. Ann Oncol 2011;22 Suppl 4:Abs 106.

18. Recher C, Coiffier B, Haioun C, et al. A prospective randomized study comparing dose intensive immunochemotherapy with R-ACVBP $v s$ standard R-CHOP in younger patients with diffuse large B-cell lymphoma (DLBCL). Groupe d'Etude des Lymphomes de l'Adulte (GELA) Study LNH032B. Blood 2010;104(ASH Annual Meeting Abstracts):Abs 109.

19. Sehn LH, Berry B, Chhanabhai M, et al. The revised International Prognostic Index (R-IPI) is a better predictor of outcome than the standard IPI for patients with diffuse large B-cell lymphoma treated with R-CHOP. Blood 2007;109:1857-61.

20. Fitoussi 0, Behaldj K, Mounier N, et al. Survival impact of rituximab combined with ACVBP and upfront consolidative autotransplantation in high risk diffuse large
B-cell lymphoma for GELA. Haematologica 2011;96:1136-43.

21. Haioun $\mathrm{C}$, Itti $\mathrm{E}$, Rahmouni $\mathrm{A}$, et al. [18F] fluoro-2-deoxy-D-glucose positron emission tomography (FDG-PET) in aggressive lymphoma: an early prognostic tool for predicting patient outcome. Blood 2005; 106:1376-81.

22. Itti E, Lin C, Dupuis J, et al. Prognostic value of interim 18F-FDG PET in patients with diffuse large B-Cell lymphoma: SUV-based assessment at 4 cycles of chemotherapy. J Nucl Med 2009;50:527-33.

23. Casasnovas R-0, Meignan M, BerrioloRiedinger A, et al. Interim [18F]fluorodeoxyglucose positron emission tomography SUVmax reduction is superior to visual analysis to predict early patient's outcome in diffuse large B-cell lymphoma. Blood 2010;104(ASH Annual Meeting Abstracts):Abs 320.

24. Marcus R, Imrie K, Belch A, et al. CVP chemotherapy plus rituximab compared with CVP as first-line treatment for advanced follicular lymphoma. Blood 2005;105:1417-23.

25. Hiddemann W, Kneba M, Dreyling M, et al. Frontline therapy with rituximab added to the combination of cyclophosphamide, doxorubicin, vincristine, and prednisone (CHOP) significantly improves the outcome for patients with advanced-stage follicular lymphoma compared with therapy with CHOP alone: results of a prospective randomized study of the German LowGrade Lymphoma Study Group. Blood 2005;106:3725-32.

26. Herold M, Haas A, Srock S, et al. Rituximab added to first-line mitoxantrone, chlorambucil, and prednisolone chemotherapy followed by interferon maintenance prolongs survival in patients with advanced follicular lymphoma: an East German Study Group Hematology and Oncology Study. J Clin Oncol 2007;25:1986-92.

27. Salles G, Seymour JF, Offner F, et al. Rituximab maintenance for 2 years in patients with high tumour burden follicular lymphoma responding to rituximab plus chemotherapy (PRIMA): a phase 3, randomised controlled trial. Lancet 2011;377: 42-51.

28. Morschhauser F, Radford J, Van Hoof A, et al. Phase III trial of consolidation therapy with yttrium-90-ibritumomab tiuxetan compared with no additional therapy after first remission in advanced follicular lymphoma. J Clin Oncol 2008;26:5156-64. 